\title{
Unified Taxonomy for Reference Ontology of Shape Features in Product Model
}

\author{
Ravi Kumar Gupta ${ }^{1,2}$ and Balan Gurumoorthy ${ }^{1}$ \\ ${ }^{1}$ CPDM, Indian Institute of Science, Bangalore -560012, India \\ ${ }^{2}$ Deptt. of Mechanical Engg., National Institute of Technology, Silchar - 788010, India \\ $\{r k g, b g m\} @ c p d m$. iisc. ernet. in
}

\begin{abstract}
This paper presents a unified taxonomy of shape features. Such taxonomy is required to construct ontologies to address heterogeneity in product/shape models. Literature provides separate classifications for volumetric, deformation and free-form surface features. The unified taxonomy proposed allows classification, representation and extraction of shape features in a product model. The novelty of the taxonomy is that the classification is based purely on shape entities and therefore it is possible to automatically extract the features from any shape model. This enables the use of this taxonomy to build reference ontology.
\end{abstract}

Keywords: Product information exchange, Shape feature taxonomy, volumetric feature, Deformation feature, Free-form surface feature.

\section{Introduction}

In product development cycle, several applications and engineering domains come into play making the ability to exchange of product data with semantics very critical. With product development happening in multiple locations with multiple computerbased tools, automatic exchange of meaning associated with product data between these tools and domains becomes important.

Ontologies have been proposed to address the issue of interoperability as they are a 'shared and common understanding of a domain that can be communicated between people and computers' [1]. A first step in constructing an ontology is the identification of scope of the ontology [2] followed by definition of formal taxonomies in the different concepts/themes that constitute the ontology [3]. These taxonomies are then linked by forming relationships between concepts across the different taxonomies. Shape entities are one of the critical concepts in any product or engineering ontology. Presently either STEP AP or vocabularies from popular CAD packages are used [3].

This paper presents a unified taxonomy of shape features to enable interoperability of heterogeneous shape models encountered during the product lifecycle. 
Shape features in a product model are (i) volumetric, (ii) deformation and (iii) freeform surface features (FFSFs). Volumetric features are the features that are associated with addition or subtraction of volume i.e. manufacturing features, design features by volume addition and/or subtraction operations. Deformation features, on the other hand, are not associated with addition or subtraction of volume but are created by deformation of material as features in sheet-metal part or by forming of material as features in injection molded part. Free-form surface features (FFSFs) are the features that modify a free-form surface in a part model.

Paper [4] has reviewed shape features extraction techniques. Nalluri [5], Han [6], Subramani [7] and Bok and Mansor [8] have reviewed shape features taxonomies for volumetric features. Liu et al., [9], Sunil and Pande [10], Gupta and Gurumoorthy [11] and Kannan and Shunmugam [12] have reviewed shape features taxonomies for sheet metal features. Pernot et al., [13], Gupta and Gurumoorthy [14] and Bok and Mansor [8] have reviewed shape features taxonomies for free-form surface features. Table 1 summarises shape features considered in the classification schemes reported in the literature. The shape features which are not handled by these classification schemes are also presented in the table. Many classification and representation schemes for representation of features and feature extraction have been developed for volumetric features. There are some approaches for deformation features [9], [10], [11], [12], [15] and free-form surface features [14], [16], [17]. Current art in feature technology does not handle the different classes of features mentioned above in a single framework to arrive at a unified representation and taxonomy.

Current art in feature technology has separate classifications for volumetric, deformation and free-form surface features. A product has all these features and therefore it is very much required to have single taxonomy for all the shape features. The paper presents unified taxonomy which allows classification, representation and extraction of shape features in a product model. This can be used for establishing semantic equivalences of shape features across heterogeneous shape models using the ontology based on this taxonomy as a reference ontology. 


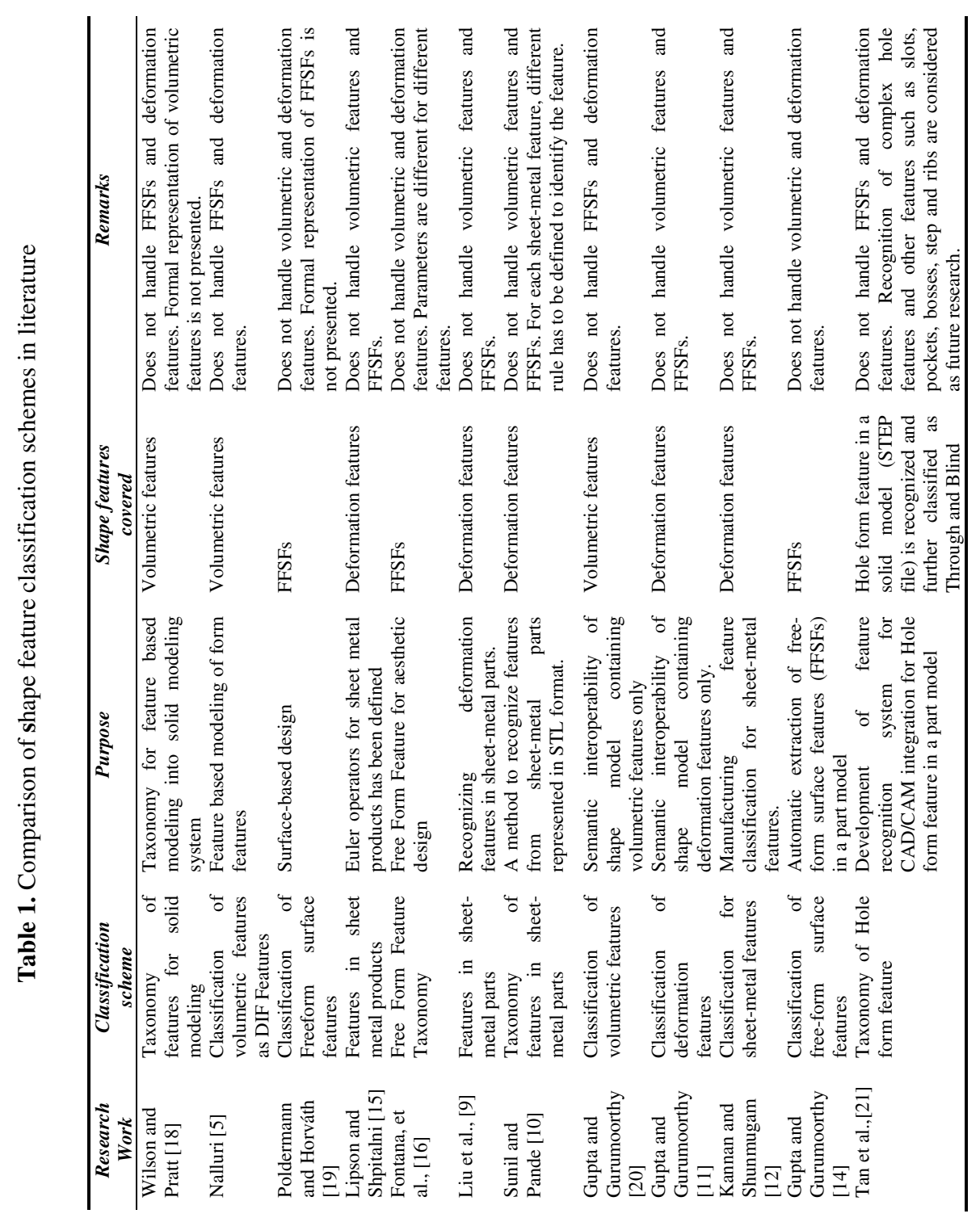




\section{Shape Features in Part Model}

A part model may have volumetric features, Deformation features and FFSFs as shown in Fig. 1. The FFSFs may be on a face of the model or on a face of volumetric feature. Example of a solid part model with volumetric features and FFSFs is shown in Fig. 1(a). Examples of volume feature, deformation features and FFSFs in a sheetmetal part are shown in Fig. 1 (b).

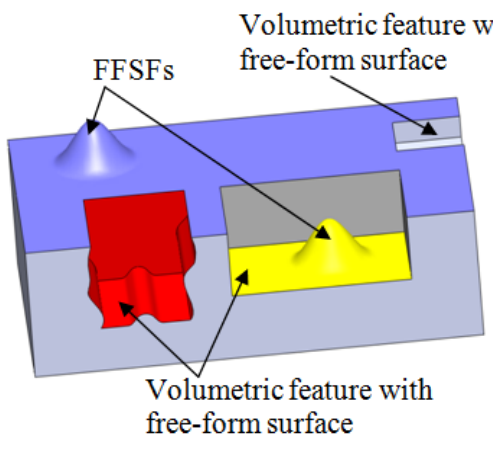

(a)

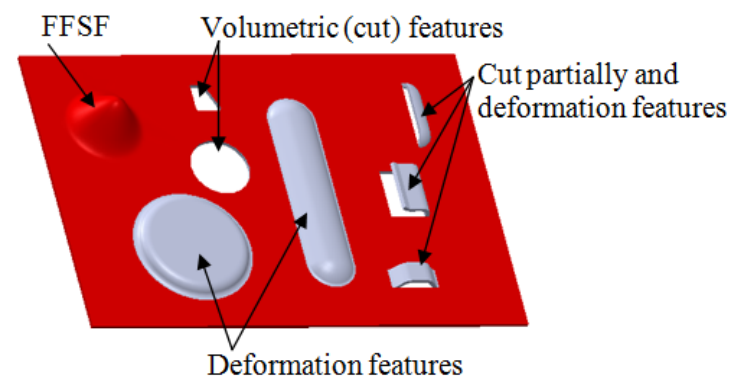

(b)

Fig. 1. Example of Shape features in part model

Shape feature is defined as a set of faces (or a face) with distinct topological and geometrical characteristics. The shape features in a product model are classified as follows:

1. Volumetric features (features created by addition or subtraction of volume),

2. Deformation features (features created by deformation of material (as in sheet-metal parts) or forming of material (as in injection molded parts of constant thickness)) - associated with constant thickness sections,

3. Free-form surface features (feature on a single face of the part model).

\subsection{Volumetric Features}

A volumetric feature is defined as a set of faces with distinct topological and geometrical characteristics and is created by the addition or subtraction of a sweep solid from an arbitrary solid [5]. The faces of the volume (solid-piece) that is added or removed are classified as shell-faces and end-faces. The faces that form the closed shell are classified as shell-faces and the faces which close the ends of the shell are classified as end-faces as shown in Fig. 2. Addition or subtraction of the solid-piece leaves an impression (feature) on the base-solid. The faces in the impression which do not exist in the base-solid before the addition or subtraction operation are classified as created faces (newly created faces). The faces in the base-solid, which are modified by the addition or subtraction operation and are shared by the solid-piece and the base-solid, are classified as shared faces (modified faces) as shown in Fig. 2. 

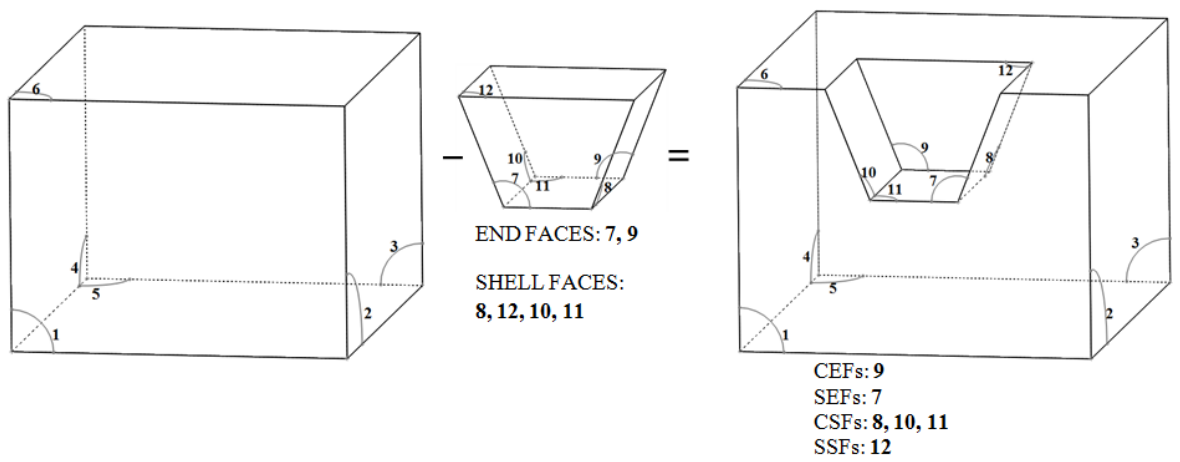

Fig. 2. Classification of feature faces for volumetric feature

The faces in the final solid associated with an individual feature are classified as follows:

Created Shell Faces (CSFs) - newly created faces in the base-solid corresponding to the shell-face of the solid-piece.

Shared Shell Faces (SSFs) - already existing faces in the base-solid corresponding to the shell-face of the solid-piece.

Created End Faces (CEFs) - newly created faces in the base-solid corresponding to the end-face of the solid-piece.

Shared End Faces (SEFs) - already existing faces in the base-solid corresponding to the end-face of the solid-piece. One shared end face of a feature can coincide with more than one face in the base-solid.

The representation of a feature in terms of these four types of faces and the characteristic relationship among them is called the Domain Independent Form Feature (DIFF) model. A classification of volumetric features based on the above classification of faces is summarized in Fig. 3.

\subsection{Free-Form Surface Features}

These features can be formed either by manipulating a free-form surface or realized during the construction of a free-form surface. The criteria for classification are based on the topology and geometry of the surface with the feature and do not rely on any domain knowledge.

The presence of a feature on a free-form surface is indicated by a distinct change in the geometric characteristics of the surface. The separating curve is the curve on the surface where this distinct change occurs so that the portion of the surface on one side of the separating curve forms the feature. This bound portion of the base surface that forms the feature is referred to as the influence region. The separating curve can be a closed or an open curve; if the separating curve is open, it will bound the feature on the surface along with a portion of the boundary curves of the base surface. 


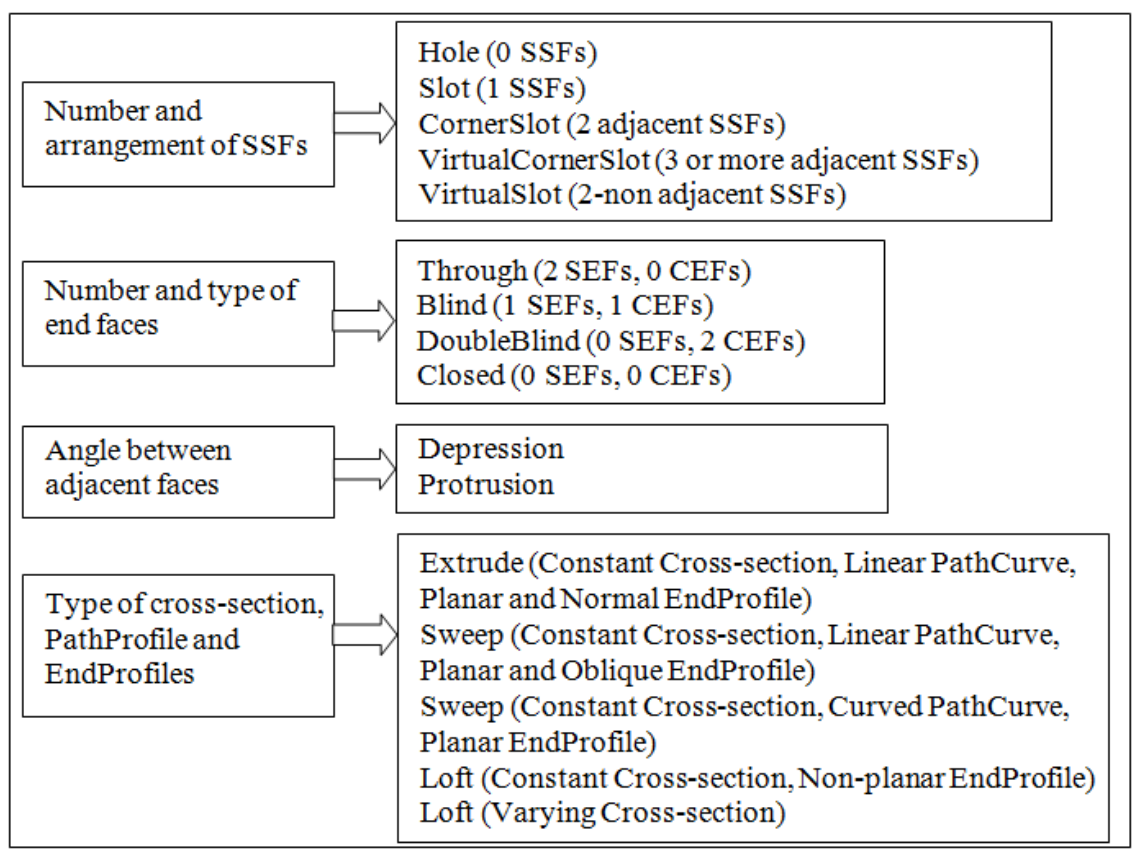

Fig. 3. Hierarchy of classification criteria in feature definition of volumetric features

\begin{tabular}{|c|c|}
\hline $\begin{array}{l}\text { Number and Type of } \\
\text { Separating Curve(s) }\end{array}$ & $\begin{array}{l}\text { DoubleBlind (1 SCs, Closed SCs), } \\
\text { Blind (1 SCs, Open SCs), } \\
\text { Through ( } 2 \text { or more SCs, Open SCs), } \\
\text { ClosedRing (2-Nested SCs, Closed SCs), } \\
\text { Closed (2-Crossing_Boundary SCs, Closed SCs) }\end{array}$ \\
\hline & Depression $([+,+]$ or $[+, 0])$, \\
\hline Signs of Principal Curvatures & $\begin{array}{l}\text { Protrusion }([-,-] \text { or }[0,-]) \text {, } \\
\text { Saddle }([+,-]) \text {, } \\
\text { Flat }([0,0])\end{array}$ \\
\hline
\end{tabular}

Fig. 4. Hierarchy of classification criteria in feature definition of FFSFs

The separating curve, its interaction with the base surface boundary and the influence region provide the criteria for classifying FFSFs. An FFSF is classified based on its type and nature. The type of an FFSF is defined in terms of the number of separating curves and the interaction of the separating curve(s) with the base surface boundary. The nature of an FFSF is decided by a combination of the signs of the principal curvatures in the influence region (the region enclosed by the separating curve). The principal curvature can be + (concave), - (convex) or 0 (neutral, flat). The number and type of separating curve(s) and the nature of principal curvatures in the influence region of each feature constitutes the DIFF model for FFSFs. The classification of FFSFs based on the above is summarized in Fig. 4. 


\subsection{Deformation Features}

Deformation features are present in constant thickness part models, as a result of deformation of material (as in sheet-metal parts) or forming of material (as in injection molded parts of constant thickness)), also referred to as constant thickness features [9].

These features are created by beading, bending, forming, folding, turning, joggling, embossing, lancing or louver operation. Example of sheet-metal deformation features are depicted in Fig. 5.

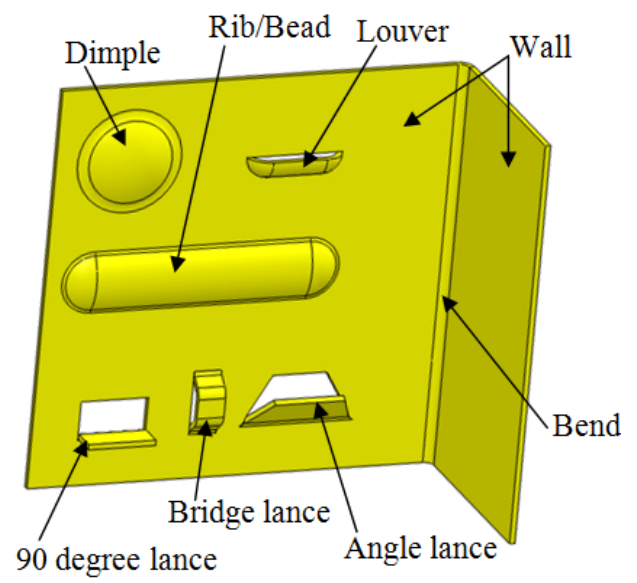

Fig. 5. Deformation features in constant thickness part

The faces in the object associated with an individual "deformation feature" are classified as follows:

Created End Faces (CEFs) - End faces in a deformation feature that are also created faces are termed as created end faces. The CEFs are further classified as Created Wall End Faces (CWEFs) and Created Bend End Faces (CBEFs) depending upon the basic deformation feature type responsible for its creation, as shown in Fig. 6(a). CBEFs are non-planar whereas CWEFs are planar.

Shared Shell Faces (SSFs) - If a shell face in a feature is a shared face then the shell face is classified as shared shell face (SSF). Examples of shared shell faces are shown in Fig. 6. The SSFs are further classified as BSSFs (Boundary SSFs) or ISSFs (Interior SSFs) based on whether the SSF of a feature is boundary shell face or interior shell face.

Created Shell Faces (CSFs) - If a shell face in a feature is created face then the shell face is classified as created shell face (CSFs) as shown in Fig. 6(a). The CSFs are further classified as BCSFs (Boundary CSFs) or ICSFs (Interior CSFs) based on whether the CSF of a feature is boundary shell face or interior shell face. 


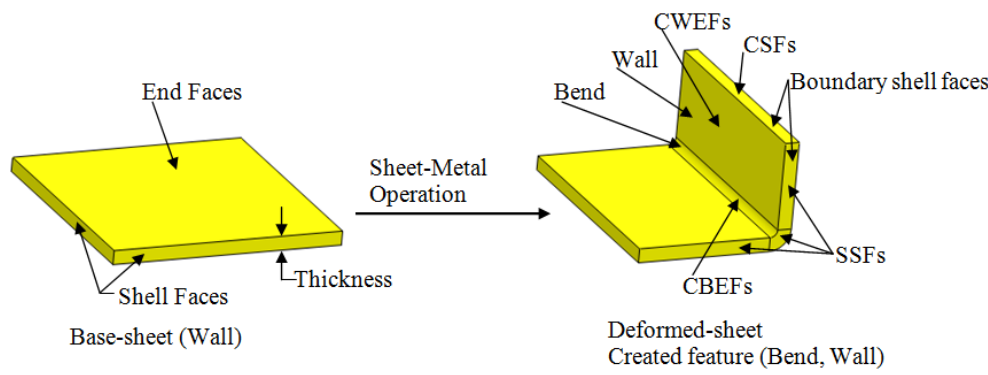

(a)

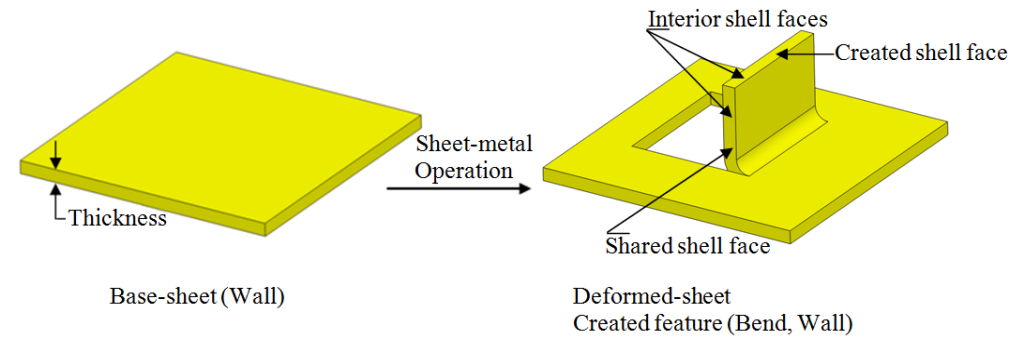

(b)

Fig. 6. Classification of feature faces for deformation features

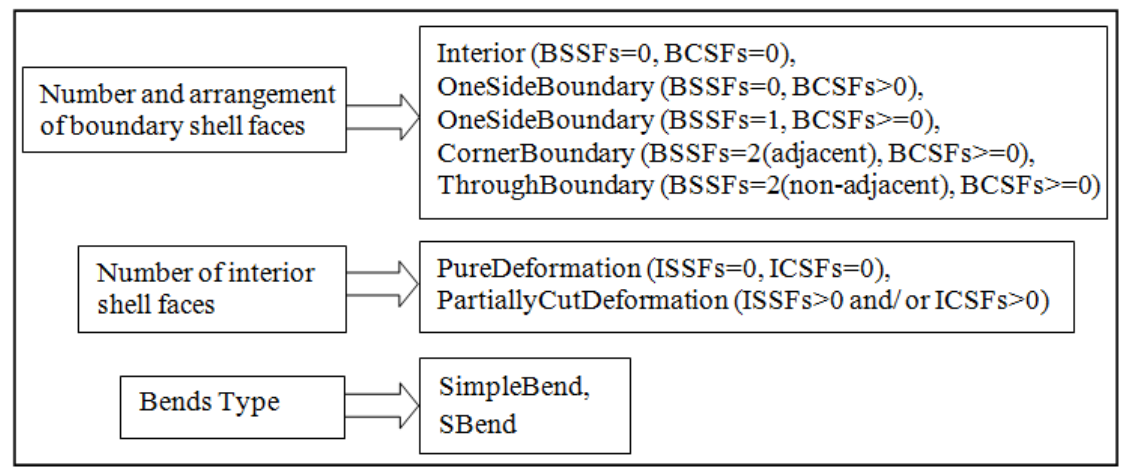

Fig. 7. Hierarchy of classification criteria in feature definition of deformation features

All features are therefore defined in terms of these six types of faces (CWEFs, CBEFs, BSSFs, ISSFs, BCSFs, ICSFs). Deformation features are represented by these six types of faces and the characteristic relationship between them in the DIFF model. The classification of deformation features based on the above classification of faces is shown in Fig. 7. 


\section{Taxonomy for Shape Features}

Based on the above classifications, for each of the three types of features, a common hierarchy of classification has been defined as shown in Fig. 8. The generic content captures information regarding general class of the feature whereas the non-generic content captures content pertaining to defining a particular instance of that general class of the feature. For the feature in Fig. 2 for instance, the generic content would pertain to the feature being a blind slot while the non-generic content would contain the detail that it is a V-shaped slot. Parameters used for classifying and defining a shape feature in the DIFF model are extracted from the geometric and topology information available in product model [5], [11], [14].

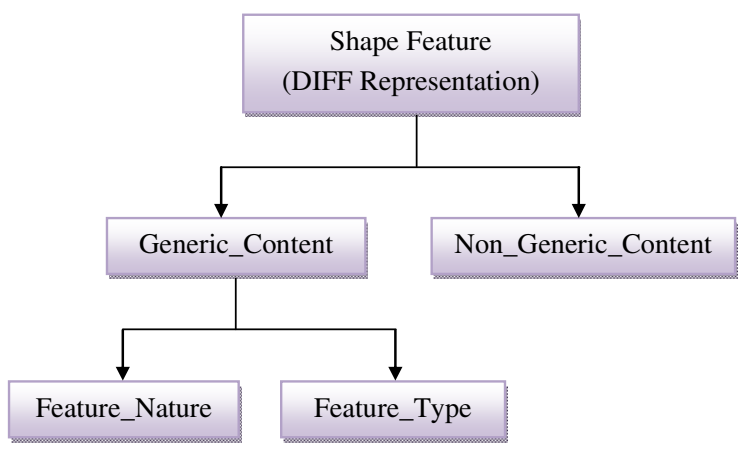

Fig. 8. Class structure of every shape feature in the DIFF model

Parameters with their possible values used for defining shape feature are shown in Figs. 3, 4 and 7 for volumetric features, free-form surface features and deformation features, respectively. These parameters are used for reasoning about shape features and are grouped under class 'Generic_Content'. These parameters are further classified and grouped as

(i) Feature_Type of a shape feature in the taxonomy is classified and represented based on the interactions of the feature with the boundary elements of the base solid/surface. Parameters associated with class 'Feature_Type' to classify shape features are based on number and arrangement of faces in a feature for volumetric and deformation features, and, number and type of separating curves in a feature for FFSFs. The differences in parameters are because of dimensionality as volumetric and deformation features are associated with volume whereas FFSFs are associated with surface.

(ii) Parameters associated with class 'Feature_Nature' to classify shape features are based on curvature characteristics in a feature. Feature_Nature of a shape feature in the taxonomy is classified and represented based on the curvature variations across entities (faces, edges) in the feature.

In the case of FFSFs, the feature is part of a base surface which has boundary edges as base surface boundary. So the interactions of the separating curves(s) with the base surface boundary curves are used to classify and define type of FFSFs, 
whereas volumetric and deformation features are part of base solid/sheet which has boundary faces as base solid/sheet boundary. So the interactions of the faces in the feature with faces in the base solid/sheet are used to classify and define the type of these features.

The feature type of a FFSF is based on a single attribute - namely the type of interaction between the separating curve and the boundary curve of the base surface. The feature type of deformation and volumetric features, on the other hand is decided by two attributes. These are (i) Number and arrangement of boundary shell faces, and (ii) Interior shell faces for deformation features, and (i) Number and arrangement of shell faces, and (ii) Number and type of end faces for volumetric features. The volumetric features are further classified based on the variations of cross-sectional shape and PathCurve in the feature. As the cross-sectional shape is constant and PathCurve is linear and normal to the EndProfile in a deformation feature, so the deformation features do not have classification based on cross-sectional shape and PathCurve. The volumetric features therefore have one more type of classification for the feature type as "Loft, Sweep and Extrude" based on the variations of the crosssectional shape and PathCurve.

The unified taxonomy for shape features is presented in Fig. 9. The taxonomy classifies shape features according to the characteristics of the parameters in the DIFF model which makes definition of a shape feature simple, consistent and amenable to automated reasoning. The number of branches for each class of feature varies with the dimensionality of space occupied by the feature, as discussed above. The proposed is referred to as a taxonomy (and not an ontology) as no associative relations across the branches or with other entities are captured [2].

\section{Discussion}

The classification schemes presented in papers [5], [7], [18], [20], [21] classify volumetric features in a part model. Deformation and freeform surface features are not discussed in these papers. The classification schemes [13], [14], [16], [17], [22], [23] do not have procedures to include volumetric and deformation features in their taxonomies. The classification schemes presented in papers [9], [10], [11], [12], [15] have classified sheet-metal features in a sheet-metal part model. The proposed unified taxonomy has all shape features in a part model, classified under single classification and representation scheme, and can be used for automated reasoning.

The reasoning module for finding semantic equivalences for exchanging volumetric features has been discussed in paper [20]. A reasoning module for finding semantic equivalences for all type of shape features and automatic construction of the product model using semantically equivalent shape features in a target application is in the development stage. The primary requirement for the reasoning module is to have unified taxonomy with formal representation for all shape features in a product model. The developed unified taxonomy with formal representation for all shape features has been presented in this paper and can be used for automated reasoning in the reasoning module. 


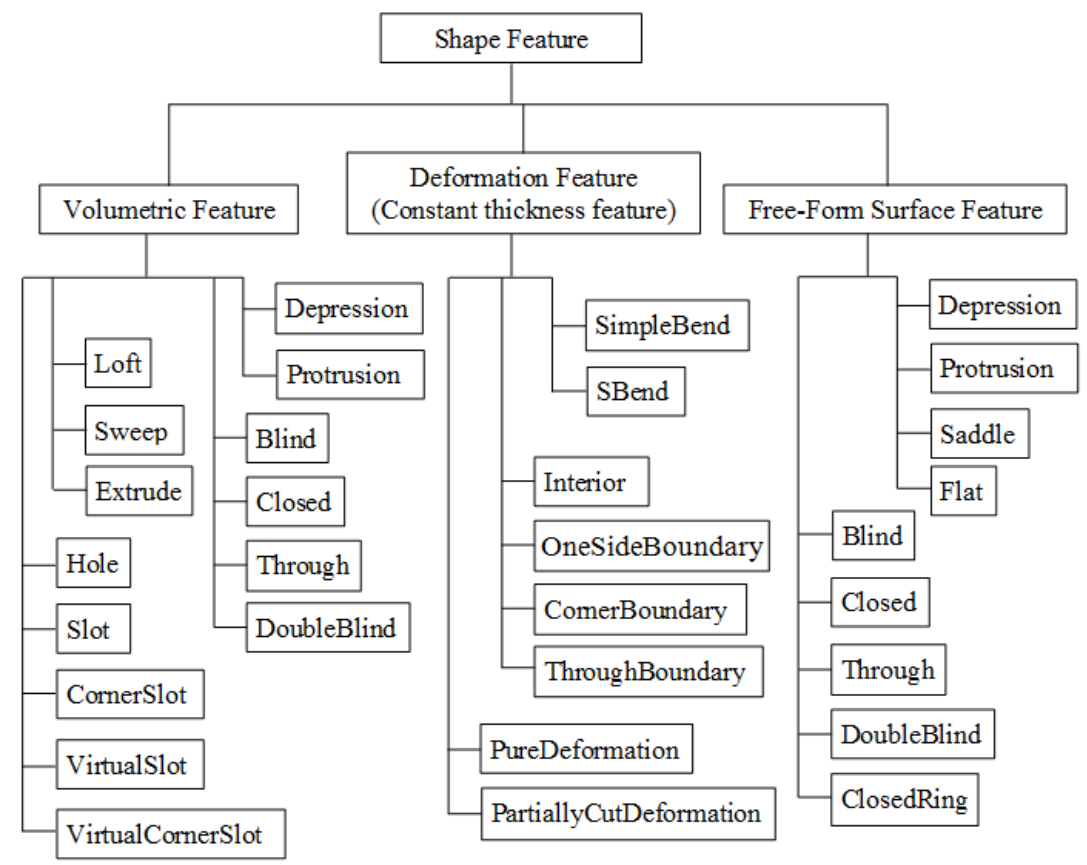

Fig. 9. Unified taxonomy for classification of shape features

The work presented in this paper brings volumetric, deformation and free-form surface features in product models under a single classification and representation. The taxonomy is unified because the basis for classification is similar (coarse at the top - nature, type and, fine at the bottom - through hole, blind slot). Classification of features in this taxonomy is concise (the maximum number of branches at any level is four and there are only three levels. As the features are classified based on the arrangement of faces/curves, the classification is purely based on form (geometry and topology) and therefore consistent. Completeness of the classification is harder to argue. At present the only claim that can be made is that the classification scheme does capture all the features that are in use in product models. Secondary features that are seen in some taxonomies are not separately classified here as, most secondary features such as fillet or blend can be modeled as a volumetric feature. The taxonomy therefore is application independent and amenable to automated reasoning.

The definition and classification thus far has been with respect to single features. In real parts features will interact and intersect. However, intersecting or interacting features do not cause a problem as long as the application does not require the interacting features to be handled as a single feature. The feature extraction routines can identify the DIF features that constitute the overlapping or interacting feature. Subsequently, the individual feature can be processed or manipulated. However, if the interacting features have to be handled as a single feature then the mapping from the DIFF based classification scheme to the application domain has to be addressed 
The classification and representation of shape features are based on faces and curves, and, a characteristic arrangement amongst them. As mentioned above, this representation uses only geometric and topological entities that are explicitly available in the shape model. Recognising these features and extracting their DIFF model automatically from a shape model is therefore feasible [5], [11], [14]. The proposed taxonomy can therefore be used to develop reference ontology for handling heterogeneity in shape models. This is because the DIFF model can be extracted from the shape model and can be used as a basis to compare different feature models of the same shape.

For any other domain specific feature, the geometry associated with the feature can be obtained unambiguously and the DIFF representation can be extracted. The parameters, required to represent a shape feature in the DIFF, can therefore be obtained from B-rep of a shape model. The extracted DIFF representation of the feature can now be used for finding semantically matching entities in the ontology based on the proposed taxonomy. Extraction of feature semantic, as understood by a target application for a matching entity, can also be obtained from the DIFF representation. Ongoing work is addressing the development of a reference ontology based on the DIFF taxonomy to enable semantic interoperability of shape models.

\section{Conclusion}

Unified taxonomy has been developed to represent, classify and extract shape features under three main classes namely, volumetric features, deformation features and freeform surface features. This taxonomy is based on the Domain Independent Form Feature (DIFF) model to represent features. Parameters in the model are geometric and topological entities available in the shape model. DIFF model of the features can therefore be automatically extracted and the features in the model are classified. The unified taxonomy is proposed as a candidate for use in defining reference ontology to handle heterogeneous shape models.

\section{References}

1. Fensel, D.: Ontologies: A silver bullet for knowledge management and electronic commerce. Springer, Berlin (2003)

2. Stevens, R., Goble, C., Bechhofer, S.: Ontology-based knowledge representation for bioinformatics. Briefings in Bioinformatics 1(4), 398-414 (2000)

3. Li, Z., Raskin, V., Ramani, K.: Developing engineering ontology for information retrieval. Trans. of ASME Journal of Computing and Information Science in Engineering 8 (2008)

4. Yang, M., Kpalma, K., Ronsin, J.: A survey of shape feature extraction techniques. In: Yin, P.-Y. (ed.) Pattern Recognition, pp. 43-90 (2008)

5. Nalluri, S.R.P.R.: Form feature generating model for feature technology. Ph.D. Thesis, Indian Institute of Science, Department of Mechanical Engineering, Bangalore, India (1994)

6. Han, J.: Survey of feature research. Technical Report IRIS-96-346, Institute for Robotics and Intelligent systems, USC, USA (1996) 
7. Subramani, S.: Feature mapping, associativity and exchange for feature-based product modeling. Ph.D. Thesis, Indian Institute of Science, Department of Mechanical Engineering, Bangalore, India (2005)

8. Bok, A.Y., Mansor, M.S.A.: Generative regular-freeform surface recognition for generating material removal volume from stock model. Computers \& Industrial Engineering 64, 162-178 (2013)

9. Liu, Z.J., Li, J.J., Wang, Y.L., Li, C.Y., Xiao, X.Z.: Automatically extracting sheet-metal features from solid model. Journal of Zhejiang University Science 5, 1456-1465 (2004)

10. Sunil, V.B., Pande, S.S.: Automatic recognition of features from freeform surface CAD models. Computer-Aided Design 40, 502-517 (2008)

11. Gupta, R.K., Gurumoorthy, B.: Extending feature based semantic interoperability for sheet-metal product development. In: Proceedings of International Conference on Trends in Product Lifecycle Modeling, Simulation and Synthesis, PLMSS 2008, Bangalore, India, pp. 236-246 (2008)

12. Kannan, T.R., Shunmugam, M.S.: Processing of 3D sheet metal components in STEP AP203 format. Part I: feature recognition system. International Journal of Production Research 47, 941-964 (2009)

13. Pernot, J.P., Falcidieno, B., Giannini, F., Léon, J.C.: Incorporating free-form features in aesthetic and engineering product design: State-of-the-art report. Computers in Industry 59, 626-637 (2008)

14. Gupta, R.K., Gurumoorthy, B.: Automatic extraction of free-form surface features (FFSFs). Computer-Aided Design 44, 99-112 (2012)

15. Lipson, H., Shpitalni, M.: On the topology of sheet metal parts. Trans. of ASME Journal of Mechanical Design 120, 10-16 (1998)

16. Fontana, M., Giannini, F., Meirana, M.: A free form feature taxonomy. In: Brunet, P., Scopigno, R. (eds.) Proceedings of Eurographics 1999, Computer Graphics Forum, vol. 18, pp. 107-118 (1999)

17. Nyirenda, P.J., Bronsvoort, W.F., Langerak, T.R., Song, Y., Vergeest, J.S.M.: A generic taxonomy for defining freeform feature classes. Computer-Aided Design \& Applications 2, 497-506 (2005)

18. Wilson, P.R., Pratt, M.J.: A taxonomy of features for solid modeling. Geometric Modeling for CAD Applications, 125-136 (1988)

19. Poldermann, B., Horváth, I.: Surface-based design based on parameterized surface features. In: Horváth, I., Varadi, K. (eds.) Proceedings of the International Symposium on Tools and Methods for Concurrent Engineering 1996, pp. 432-446. Institute of Machine Design, Budapest (1996)

20. Gupta, R.K., Gurumoorthy, B.: A feature-based framework for semantic interoperability of product models. SV - Journal of Mechanical Engineering 54, 446-457 (2008)

21. Tan, C.F., Kher, V.K., Ismail, N.: Design of a feature recognition systems for CAD/CAM integration. World Applied Sciences Journal 21(8), 1162-1166 (2013)

22. Nyirenda, P.J., Bronsvoort, W.F.: Numeric and curve parameters for freeform surface feature models. Computer-Aided Design 40, 839-851 (2008)

23. Nyirenda, P.J., Mulbagal, M., Bronsvoort, W.F.: Definition of Freeform Surface Feature Classes. Computer-Aided Design \& Applications 3, 665-674 (2006) 